\title{
The Formation of Alveoli in Rat Lung during the Third and Fourth Postnatal Weeks: Effect of Hyperoxia, Dexamethasone, and Deferoxamine
}

\author{
LUCIANO N. BLANCO AND LEE FRANK \\ Pulmonary Research Center, Departments of Medicine and Pediatrics, University of Miami School of Medicine, \\ Miami. Florida 33101
}

\begin{abstract}
Terminal gas-exchange units in the lung of many species are, at birth, relatively large structures termed saccules. Saccules septate postnatally forming smaller units that constitute the final alveoli. In the rat, septation occurs intensively during the first 2 postnatal wk after which it has been considered to stop. Treatment with dexamethasone or exposure to hyperoxia during the first 2 postnatal wk markedly inhibits septation as evidenced by the formation of fewer and bigger alveoli than in normally developed rats. Deferoxamine, an iron chelator, has been reported to protect the lung from the effects of exposure to hyperoxia in early postnatal life. In this study, we investigated the effects of these treatments during the 3rd and 4th postnatal wk, that is, after the early period of rapid alveolarization. Our results show that treatment with dexamethasone no longer had any inhibitory effect on alveoli formation; that exposure to hyperoxia continued to inhibit the formation of new alveoli, resulting in bigger and less numerous alveoli; that treatment of animals exposed to hyperoxia with deferoxamine still protected their lungs against hyperoxic inhibition; and that elastin fiber length density in the lung was significantly reduced in hyperoxicexposed animals. These results suggest that septation continues beyond the 2nd postnatal wk and does not stop abruptly at age $14 \mathrm{~d}$ in air-breathing rats and that hyperoxic inhibition of alveolarization during the 3rd and 4th postnatal wk is due to the inhibition of septation of existing or newly generated gas-exchange units during that period of lung development. (Pediatr Res 34: 334-340, 1993)
\end{abstract}

Abbreviations

DEX, dexamethasone

DFX, deferoxamine

HYP, hyperoxia

To provide sufficient oxygenation according to the increasing metabolic needs of the animal as it grows, the lung requires a mechanism or mechanisms to increase the surface area of its gasexchange region. In humans and rats, as in many other species, this enlargement of the gas-exchange surface area is achieved primarily by the postnatal formation of alveoli, the basic gasexchange unit in the mature lung. At birth, the terminal air units are relatively large and relatively thick walled and at this stage are termed saccules $(1,2)$. Saccules septate (subdivide) postna-

Received July 27, 1992; accepted April 9, 1993.

Correspondence: Luciano N. Blanco, Ph.D., Pulmonary Research Center (R. 120). University of Miami School of Medicine, P.O. Box 016960, Miami, FL 33101 . tally forming smaller units that after a process of wall thinning constitute the final alveoli $(2,3)$. In rats, septation starts about the 3 rd or 4 th postnatal $\mathrm{d}$ and has generally been considered completed by the end of the 2 nd postnatal wk (4), although there is inconclusive evidence about the end point, and it has been postulated that septation might indeed continue up to adulthood (5-6). In humans, it is now generally believed that alveoli formation is largely completed by around age $2 \mathrm{y}$, rather than extending to age $8 \mathrm{y}$ as previously believed (7).

Surface area of any structure can be altered by four distinct means: expansion (change in volume), subdivision (septation), replication, or change of shape (without change in volume). In principle, the growing lung could use all these mechanisms to provide the necessary enlargement of the gas-exchange surface area, and each one of them could be expected to be regulated in a different way. Septation of the saccules present at birth has been considered the mechanism primarily responsible for the rapid several-fold increase in surface area of the gas-exchange region during the first 2 postnatal wk. Daily treatment of rats with DEX during early postnatal life ( 3 to $13 \mathrm{~d}$ ) has been shown to inhibit the formation of alveoli during this period of rapid alveoli formation $(8,9)$. Exposure to HYP during the first 2 postnatal wk also has been shown to produce similar inhibitory effects (10). In both cases, the inhibition seems to occur by blocking septation of the immature large saccules present at, or formed after, birth. Inhibition of septation produces larger and fewer alveoli than in normally developed untreated rats (9). During the first 2 postnatal wk, HYP has been shown to also decrease elastin fiber length density (11). The effect of DEX treatment or hyperoxic exposure during this early postnatal period of normal alveolarization seems to be a permanent one, with larger and fewer alveoli persisting into adulthood (8-12). DFX, an iron chelator, has been reported to protect the lung against the hyperoxic inhibition of alveolarization during the first 2 postnatal wk (12).

After the 2nd postnatal wk as the animal continues to grow, the surface area of the gas-exchange region of the lung continues to grow, matching the metabolic needs of the animal. To explain this later increment in gas-exchange surface area, many investigators have postulated the expansion of the existing alveoli at the end of the 2 nd postnatal wk $(1,13)$. Others have assumed the formation of new alveoli by yet unidentified means (8) or, recently, by the continued formation of new saccules and their subsequent septation up to adulthood (5).

In this study, we investigated the effect of HYP, DEX, and DFX after the early period of rapid lung alveolarization. We treated, and/or exposed animals to these agents from postnatal d 18 to d 28 in an effort to elucidate the mechanisms of later alveoli formation and also to determine whether the more mature lung is still susceptible to the potential inhibitory effects of these common therapeutic agents. 


\section{MATERIALS AND METHODS}

Animals. We used adult Sprague-Dawley albino rats as breeders (Charles River Laboratories, Portage, MI). Pups and mother rats were from our own long-term rat breeding program. They were housed in the Animal Care Facilities of the University of Miami School of Medicine under veterinary supervision, maintained on regular laboratory food and water ad libitum, and kept on a 12-h light/dark cycle. National Institutes of Health guidelines for humane animal treatment were followed in all experimental procedures, and the protocol was preapproved by the University Animal Care Committee.

Experimental Conditions. Pups were maintained with their dams in room air until postnatal d 18. On this day for each experiment, pups from six litters were pooled and randomly divided among the dams ( 10 to 12 per litter). A 10-d treatment and exposure period began by injecting the pups s.c. with either DEX $(2 \mu \mathrm{g} / \mathrm{kg} / \mathrm{d})$, DFX $(250 \mathrm{mg} / \mathrm{kg} / \mathrm{d})$, or equal volume of saline, using an injection volume of $5 \mu \mathrm{L} / \mathrm{g}$ of body mass. One half of the treated animals were exposed continuously to $>95 \%$ oxygen, and one half were maintained in room air. Dams were switched between oxygen and air litters every $24 \mathrm{~h}$ to prevent oxygen toxicity in the dams and resulting poor nurturing. Oxygen exposures were conducted as previously described $(12,14)$ in 3.5 $\mathrm{ft}^{3}$ plastic isolettes with regular monitoring of oxygen $(>95 \%)$ and carbon dioxide $(<0.40 \%)$, temperature $\left(23\right.$ to $\left.25^{\circ} \mathrm{C}\right)$ and humidity (40 to $60 \%$ ). Exposures were continuous except for a daily 20 -min to $30-\mathrm{min}$ interval to allow for cage changing, dam switching, and pup weighing and injection. Rats were killed at postnatal d 28 just at the end of the 10-d experimental period (five rats per treatment, for a total of 25 rats). In another set of experiments, normal untreated rat pups in room air were killed at postnatal $\mathrm{d} 13$ (three rats).

Alveolar Morphometry. Lung fixation. Rats were anesthetized with an overdose of pentobarbital sodium $(60 \mathrm{mg} / \mathrm{kg}$, intraperitoneally), and when unresponsive to toe pinch, their tracheas were rapidly cannulated through a midline cervical incision while they were still breathing. A bilateral pneumothorax was produced by puncturing the diaphragm from its abdominal surface, and cold $2.5 \%$ glutaraldehyde in $0.1 \mathrm{M}$ sodium cacodylate buffer $(\mathrm{pH}$ 7.4) was infused into the trachea at a gauge pressure equivalent to $20 \mathrm{~cm} \mathrm{H}_{2} \mathrm{O}$ (approximately $1960 \mathrm{~Pa}$ ). When flow ceased, the trachea was ligated and the lungs were removed from the thorax and immersed in fixative of the same composition as had been used to inflate the lung. Fixation was continued for $2 \mathrm{~h}$ at $4^{\circ} \mathrm{C}$, and lung vol was determined by volume displacement (15).

Sampling and preparation of tissue. Tissue blocks were selected using an area-weighted sampling technique (16). Each lung was cut into approximately $0.3-\mathrm{cm}^{2}$ slices that were placed randomly over a grid containing circles $3.5 \mathrm{~mm}$ in diameter at a spacing of $1 \mathrm{~cm}$. A minimum of 10 blocks (approximately $1 \mathrm{~mm}^{3}$ ) per lung were cut from sites where the slices overlaid the circles. The selected blocks were fixed, dehydrated, and embedded in Poly/ Bed 812 as previously described (9).

Stereology. Approximately 120 to 150 serial sections per block were obtained with a diamond knife at approximately $1.0-\mu \mathrm{m}$ thickness on a Sorvall MT-2 ultramicrotome. Each group of serial sections covered a depth of about $150 \mu \mathrm{m}$. The sections were mounted on glass slides in the proper sequence, stained with toluidine blue, and photographed with a Zeiss microscope on 35-mm Ektachrome film. The photographic slides were then transilluminated on a light box for analysis, and those chosen for measurements were projected on a suitable test system (see below) at a final magnification of $\times 1000$.

Serial sections allowed unambiguous identification of alveoli and alveolar ducts by a three-dimensional analysis of the structures. An alveolus was defined as a gas-exchange structure with a mouth in communication with a common air space identified as an alveolar duct. Small structures opening into an alveolus and, therefore, not in direct communication with an alveolar duct, were considered part of the alveolus itself and not an independent structure. The mouth of each structure was defined by an imaginary straight line closing the opening of the structure into the alveolar duct.

Lung volume was divided into four compartments: alveolar air, alveolar duct air, gas-exchange tissue (alveolar walls), and conducting structures (airways and blood vessels). Volume density of alveolar air, alveolar duct air, gas-exchange tissue, and conducting structures, taking the whole lung as reference volume. were determined by point counting (17). Arbitrarily selected sections from the group of serial sections not too close to the beginning or end of the series of sections so as to be able to identify all structures were projected on a test system containing 150 points $1.9 \mathrm{~cm}$ apart. Points falling on each class of structure being determined were counted and divided by the total number of points representing the reference space. According to the procedure outlined in Reference 17, we count enough points and sections aiming at a relative standard error of $10 \%$ or less.

Point and intersection counting (17) was used to estimate total gas-exchange surface area and total area of alveolar mouths. Using the same test system described above and projecting on it the previously selected sections for the above mentioned measurements, we counted intersections (I) of the lines of the test system with either gas-exchange tissue (tissue-air profile boundary) to estimate total gas-exchange surface area, or with a straight line drawn to close the mouth of each structure identified as an alveolus to estimate total mouth surface area. In each case, the surface area $(\mathrm{S})$ was calculated from the expression:

$$
\mathrm{S}=2 \mathrm{I} \cdot \mathrm{V}_{\mathrm{L}} / \mathrm{P} \cdot \mathrm{d}
$$

where $V_{L}$ represents lung volume, $P$ is the total number of points corresponding to the reference space, and $d$ is the distance between points on the test system (17).

To estimate the volume of the average alveolus and alveolar number, alveoli were first selected using the selector (disector of unknown thickness) method (18), which allows the structures to be chosen with equal probability irrespective of their size, shape, or orientation.

Each stack of sections was roughly divided into three parts. In the top third, a look-up plane was selected on a section as close as possible to the first one. A reference plane with a defined counting frame was then chosen on a section at a distance approximately equal to one third of the stack height (somewhat less than the maximum mean caliper diameter of the structures). This set of plane and the space between them identified one selector. A second selector was then defined in a similar manner using the bottom third of the stack with the look-up plane chosen close to the last section of the stack. The idea was to maximize the number of particles selected in each stack while at the same time avoid as much as possible a given particle being sampled by both reference planes simultaneously. The position of the look-up and reference sections was established with independence of the position of the alveoli. Once the position of the sections was established, the three-dimensional analysis of the stack was begun to identify alveoli and alveolar duct air spaces. Alveoli that appear on a reference plane within the defined counting frame but did not appear on the look-up plane were selected for volume determination. Alveoli found completely contained between the reference plane and the look-up plane and with their projection on the reference plane within the counting frame were also selected. The volume of each selected structure (using the selector) was then estimated by means of the point-sampled intercepts method (when applied to structures previously selected with equal probability, this method allows the estimation of the number-weighted mean alveolar volume rather than volume-weighted mean volume that is obtained when the method is applied on random sections) $(18,19)$. For this purpose, each section (slide) comprising the selector and containing the relevant transects of the selected alveoli was projected on a test system consisting of parallel lines with test points. The 
position and orientation of the test system were determined following two sets of random numbers. Each transection corresponding to the same alveolus hit by a test point was classified with an arithmetic ruler, the corresponding segment cubed, and their average used to estimate the individual volume of the selected alveolus. The volume of the average alveolus was obtained from the arithmetic means of the individual estimated volumes. Because the method to estimate the volume was applied to structures previously selected irrespective of their volume, shape, or orientation, the method allows the estimation of the number-weighted, rather than volume-weighted, mean alveolar volume. The total number of alveoli per lung $(N)$ was calculated using the following equation:

$$
\mathrm{N}=\mathrm{V}_{\mathrm{L}}, \mathrm{Vv}_{\mathrm{a}} / \mathrm{V}_{\mathrm{a}}
$$

where $V_{L}$ is the lung volume, $V_{V_{a}}$, is the volume density of alveoli, and $V_{a}$ is the number-weighted mean alveolar volume.

All determinations were done on postfixed, dehydrated, and embedded tissue with the exception of total lung volume, which was obtained by fluid displacement in fixed but not dehydrated and embedded material. Because of this disparity, we determined the shrinkage factor of the tissue from immediately before postfixation to the completion of tissue embedding and found a linear shrinkage factor of $4.25 \pm 0.35 \%$. However, our values have not been corrected for shrinkage because this factor was considered not to have any essential influence on the differences of the relevant parameters we have found and also to facilitate comparison with previous studies.

Our sampling design allowed us to obtain SD smaller than the absolute differences we tried to detect between groups ( 25 to $30 \%$ or more) and coefficients of error of $10 \%$ or less.

Elastin fiber length density has been shown to be altered by HYP exposure during the first 2 postnatal wk (11). Estimation of elastin fiber length density was not included in the original protocol of this study. It was decided to include it here at a later stage when it was realized that septation seems present after the 2nd postnatal wk and possibly inhibited by HYP in an effort to investigate the effect of HYP on elastin fibers after the 2nd postnatal wk. For this reason, we used a different sampling procedure for this determination.

After killing the rats with an intraperitoneal overdose of pentobarbital, another group of 28-d-old experimental rats (five per group) had their lungs fixed in situ with $10 \%$ buffered formalin,

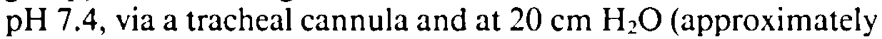
$1960 \mathrm{~Pa}$ ) (fixative) pressure. After $24 \mathrm{~h}$ fixation, lung sections randomly obtained from similarly oriented areas of the left, right middle, and right lower lobes were embedded in paraffin and processed for orcein staining. To estimate the relative length density of elastin fibers in lung parenchyma, a technique for length density measurements was used $(17,20)$.

Coded sections were observed under the microscope at $\times 400$, magnification using an ocular test system consisting of seven parallel lines equally spaced. The test system was much smaller than the total section area, allowing the observation of 30 random fields per section. Each line of the test system represented a plane perpendicular to the plane of the section. The section was screened parallel to each of the seven lines, and the number of intersections of the lines with the projected images of black elastin fibers $(<1 \mu \mathrm{m}$ diameter) was recorded. Each of these intersections corresponds to a fiber transected by the test plane. Length density $\left(\mathrm{L}_{v}\right)$ can be estimated by the expression $\mathrm{L}_{\mathrm{v}}=$ $2 \mathrm{P}_{\mathrm{A}}$, where $\mathrm{P}_{\mathrm{A}}$ is number of transection per unit area of test plane. However, no attempt was made to quantitatively estimate the dimension of the test plane perpendicular to the plane of the section (approximately $5 \mu \mathrm{m}$ ), but because this was constant for all sections scanned, the number of transections yielded relative values rather than absolute values of length density. Thirty random fields per lung were counted (omitting fields in which large vessels or airways with circumferential elastin fibers were present), and the mean number of transections per lung field was calculated for each coded slide. It is to be noted that elastin fibers do not exhibit any anisotrophy in the lung tissue.

Statistics. Groups were statistically compared by one-way analysis of variance, Duncan's multiple range test (21), and Kramer's extension of Duncan's test (22), with $p<0.05$ considered a significant difference between group mean values.

\section{RESULTS}

Table 1 shows the volume densities of alveolar air, alveolar duct air, and gas-exchange tissue. At $28 \mathrm{~d}$, alveolar air normally amounts to approximately $50 \%$ of the total lung volume, alveolar duct air to about 25 to $30 \%$ and gas-exchange tissue to approximately $20 \%$. Conducting structures (not shown) represent the remaining approximately $5 \%$ of the lung volume. There were no significant differences between treatments or ages for any of these lung fractions.

Volumes $(\mathrm{mL})$ for whole lung, alveolar air, alveolar duct air, and gas-exchange tissue are shown in Table 2 . At age $28 \mathrm{~d}$, these volumes are about 2.2- to three-fold the values found at age 13 d. There were significant differences, as expected, for all these categories between the ages of $13 \mathrm{~d}$ and $28 \mathrm{~d}$ (at $p<0.01$ level) except for gas-exchange tissue of animals $13 \mathrm{~d}$ of age versus AirDEX $28 \mathrm{~d}$ of age (not significant) and versus Air-Saline $28 \mathrm{~d}$ of age $(p<0.05)$. At age $28 \mathrm{~d}$, the only significant differences found were for whole lung volume, alveolar air, and gas-exchange tissue $(p<0.05)$ between Air-DEX and HYP-DEX, with values about 25 to $30 \%$ higher in HYP-DEX compared with Air-DEX.

Table 3 contains the values found for total gas-exchange surface area and total mouth surface area. A significant difference $(p<0.01)$ was found only between the ages of $13 \mathrm{~d}$ and $28 \mathrm{~d}$ for both categories, with values about 2.2- to 2.5 -fold higher at 28 d.

Total number of alveoli per lung and volume of the average alveolus for all experimental conditions are shown in Table 4 Significant differences were found $(p<0.01)$ between d 13 and d 28 Air-Saline, Air-DEX, and HYP-DFX for number of alveoli, with values ranging between 50 and $120 \%$ higher at $\mathrm{d} 28$, and between $\mathrm{d} 13$ and $\mathrm{d} 28 \mathrm{HYP}$-Saline and HYP-DEX for volume of the average alveolus, with values at $\mathrm{d} 28$ ranging between 20 and $100 \%$ higher than at $\mathrm{d} 13$. Between treatments at $\mathrm{d} 28$, significant differences found for number of alveoli and volume of the average alveolar $(p<0.05)$ were for Air-Saline, Air-DEX, and HYP-DFX versus HYP-Saline and HYP-DEX. The HYP groups (HYP-Saline and HYP-DEX) had approximately $30 \%$ fewer alveoli than the normal (Air-Saline) animals at age $28 \mathrm{~d}(p)$ $<0.05$ ), and DFX prevented this reduction in alveoli number during hyperoxic exposure. Similarly, mean alveolar volume was increased approximately $50 \%$ in the hyperoxic animal lungs (HYP-Saline and HYP-DEX) $(p<0.05)$, and DFX treatment blocked the hyperoxic effect. DEX treatment of animals maintained in room air had no effect on either alveoli number or volume of the average alveolus.

As shown in Figure 1, relative length density of elastin fiber

Table 1. Volume densit,*

\begin{tabular}{lcccc}
\hline & & \multicolumn{3}{c}{ Volume density } \\
\cline { 3 - 5 } \multicolumn{1}{c}{ Condition } & $\begin{array}{c}\text { Age } \\
\text { (d) }\end{array}$ & $\begin{array}{c}\text { Alveolar } \\
\text { air }\end{array}$ & $\begin{array}{c}\text { Alveolar } \\
\text { duct air }\end{array}$ & $\begin{array}{c}\text { Gas-exchange } \\
\text { tissue }\end{array}$ \\
\hline Air $(n=3)$ & 13 & $0.42 \pm 0.04$ & $0.26 \pm 0.06$ & $0.18 \pm 0.02$ \\
Air-Saline $(n=5)$ & 28 & $0.49 \pm 0.01$ & $0.30 \pm 0.02$ & $0.18 \pm 0.02$ \\
Air-DEX $(n=5)$ & 28 & $0.48 \pm 0.02$ & $0.30 \pm 0.03$ & $0.17 \pm 0.02$ \\
HYP-Saline $(n=5)$ & 28 & $0.46 \pm 0.01$ & $0.29 \pm 0.02$ & $0.22 \pm 0.01$ \\
HYP-DEX $(n=5)$ & 28 & $0.46 \pm 0.02$ & $0.30 \pm 0.02$ & $0.21 \pm 0.03$ \\
HYP-DFX $(n=5)$ & 28 & $0.44 \pm 0.03$ & $0.34 \pm 0.03$ & $0.20 \pm 0.01$ \\
\hline
\end{tabular}

* Values are mean \pm standard error. DEX, treated with DEX $(2 \mu \mathrm{g} /$ $\mathrm{kg} / \mathrm{d}$ ); HYP, HYP-exposed animals from d 18 to d 28; DFX, treated with $\operatorname{DFX}(250 \mathrm{mg} / \mathrm{kg} / \mathrm{d})$; saline animals received equivalent injections of diluent daily. 
Table 2. V'olumes*

\begin{tabular}{|c|c|c|c|c|c|}
\hline \multirow[b]{2}{*}{ Condition } & \multirow[b]{2}{*}{ Age (d) } & \multicolumn{4}{|c|}{ Volume (mL) } \\
\hline & & Lung & Alveolar air & Alveolar duct air & Gas-exchange tissue \\
\hline $\operatorname{Air}(n=3)$ & 13 & $1.18 \pm 0.03 \dagger$ & $0.50 \pm 0.04 \dagger$ & $0.30 \pm 0.07 \dagger$ & $0.22 \pm 0.02 \ddagger$ \\
\hline Air-Saline $(n=5)$ & 28 & $2.82 \pm 0.20$ & $1.39 \pm 0.09$ & $0.83 \pm 0.06$ & $0.52 \pm 0.08$ \\
\hline Air-DEX $(n=5)$ & 28 & $2.59 \pm 0.17 \S$ & $1.24 \pm 0.08 \$$ & $0.76 \pm 0.08$ & $0.46 \pm 0.09 \$$ \\
\hline HYP-Saline $(n=5)$ & 28 & $3.08 \pm 0.21$ & $1.42 \pm 0.09$ & $0.90 \pm 0.09$ & $0.68 \pm 0.05$ \\
\hline HYP-DEX $(n=5)$ & 28 & $3.38 \pm 0.21$ & $1.53 \pm 0.05$ & $1.02 \pm 0.10$ & $0.73 \pm 0.11$ \\
\hline HYP-DFX $(n=5)$ & 28 & $3.13 \pm 0.22$ & $1.36 \pm 0.10$ & $1.07 \pm 0.16$ & $0.61 \pm 0.02$ \\
\hline
\end{tabular}

* Values are mean \pm standard error.

$\dagger p<0.01$ us all age $28 \mathrm{~d}$ group values.

$\ddagger p<0.01$ is HYP-Saline, HYP-DEX, and HYP-DFX; $p<0.05$ is Air-Saline.

$\S p<0.05$ is HYP-DEX.

Table 3. Gas-exchange surface area and mouth surface area*

\begin{tabular}{lccc}
\hline \multicolumn{1}{c}{ Condition } & $\begin{array}{c}\text { Age } \\
\text { (d) }\end{array}$ & $\begin{array}{c}\text { Gas-exchange } \\
\text { surface area } \\
\left(\mathrm{cm}^{2}\right)\end{array}$ & $\begin{array}{c}\text { Mouth } \\
\text { surface area } \\
\left(\mathrm{cm}^{2}\right)\end{array}$ \\
\hline Air $(n=3)$ & 13 & $675 \pm 74 \dagger$ & $240 \pm 38 \dagger$ \\
Air-Saline $(n=5)$ & 28 & $1711 \pm 69$ & $664 \pm 51$ \\
Air-DEX $(n=5)$ & 28 & $1596 \pm 89$ & $597 \pm 19$ \\
HYP-Saline $(n=5)$ & 28 & $1536 \pm 78$ & $654 \pm 51$ \\
HYP-DEX $(n=5)$ & 28 & $1659 \pm 53$ & $633 \pm 20$ \\
HYP-DFX $(n=5)$ & 28 & $1732 \pm 105$ & $675 \pm 56$ \\
\hline
\end{tabular}

* Values are mean \pm standard error.

$\dagger p<0.01$ is all age $28 \mathrm{~d}$ group values.

Table 4. Number of alveoli and volume of average alveolus*

\begin{tabular}{lccc}
\hline \multicolumn{1}{c}{ Condition } & $\begin{array}{c}\text { Age } \\
(\mathrm{d})\end{array}$ & $\begin{array}{c}\text { Number of } \\
\text { alveoli } \times 10^{-6}\end{array}$ & $\begin{array}{c}\text { Vol of average } \\
\text { alveolus } \times 10^{-4} \mu \mathrm{m}^{3}\end{array}$ \\
\hline Air $(n=3)$ & 13 & $20.5 \pm 4 \dagger$ & $2.56 \pm 0.4 \ddagger$ \\
Air-Saline $(n=5)$ & 28 & $42.3 \pm 3 \S$ & $3.39 \pm 0.4 \S$ \\
Air-DEX $(n=5)$ & 28 & $42.2 \pm 4 \S$ & $3.11 \pm 0.5 \S$ \\
HYP-Saline $(n=5)$ & 28 & $30.2 \pm 2$ & $5.03 \pm 0.5$ \\
HYP-DEX $(n=5)$ & 28 & $31.5 \pm 3$ & $5.00 \pm 0.5$ \\
HYP-DFX $(n=5)$ & 28 & $44.8 \pm 5 \S$ & $3.22 \pm 0.5 \$$ \\
\hline
\end{tabular}

* Values are mean \pm standard error.

$\dagger p<0.01$ vs Air-Saline, Air-DEX, and HYP-DFX.

$\ddagger P<0.01$ is HYP-Saline and HYP-DEX.

$\S p<0.05$ is HYP-Saline and HYP-DEX.

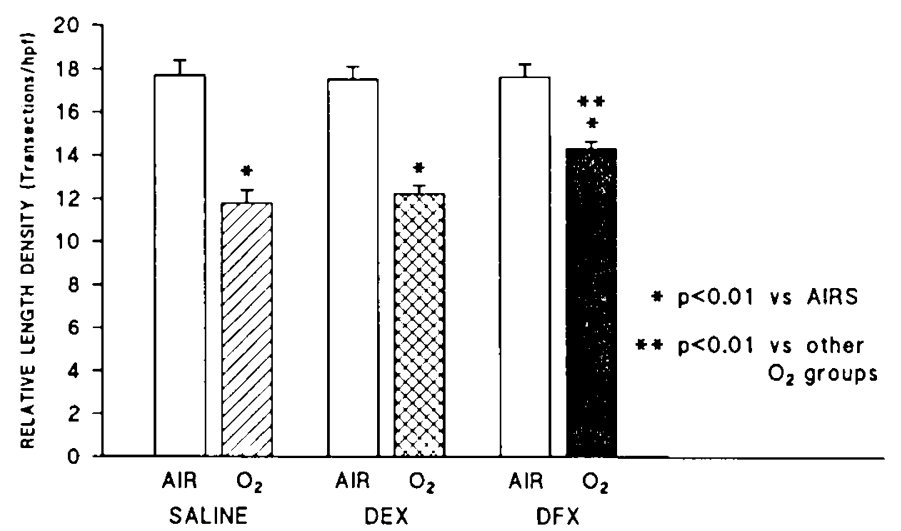

Fig. 1. Relative lung elastin fiber length density in 28-d-old rat lungs. Orcein-stained elastin fibers were quantitated in lung sections from airexposed and $>95 \%$ oxygen-exposed treated and untreated animal groups at $28 \mathrm{~d}$. Values are mean ( $\pm \mathrm{SD}$ ) number of transections per high-power field $(\times 400)$ using superimposed seven-line grid test system and examining coded sections from 30 random fields per slide (five lung slides/ group). DEX, daily treatment ( $2 \mu \mathrm{g} / \mathrm{kg} / \mathrm{d})$ from d 18 to $\mathrm{d} 28$ while in air or HYP; DFX, daily treatment with $250 \mathrm{mg} / \mathrm{kg} / \mathrm{d}$. was significantly reduced by HYP in the oxygen-exposed control pup lungs (HYP-Saline) compared with the air control group $(-33 \%, p<0.01)$. DEX treatment, in contrast, had no effect on elastin fiber deposition in the developing lungs as assessed by transection counting, either in air versus the air control, or in oxygen versus the oxygen control group. DFX treatment of the oxygen exposed rats partially reversed the marked inhibitory effect of HYP on normal lung elastin development $(-19 \%$ versus air controls; $p<0.01$ versus oxygen control group value). In addition to the significant decreases in the relative length density of elastin fibers in the HYP-exposed groups, many elastin fibers appeared qualitatively less dense and often had an abnormal frayed appearance. The light micrographs (Fig. 2) demonstrate these findings.

\section{DISCUSSION}

DEX administered postnatally from $\mathrm{d} 3$ after birth to $\mathrm{d} 13$ has been shown to markedly inhibit septation as evidenced by significantly larger and less numerous alveoli than those found in normal rats $(8,9)$. This effect seems to be irrevocable, as the lung does not recuperate (even by adulthood, age $60 \mathrm{~d}$ ) by generating smaller and more numerous alveoli after the treatment is discontinued at the end of the 2 nd postnatal wk $(8,9)$. In the present study, we showed evidence that if $10 \mathrm{~d}$ of treatment with DEX is initiated at d 18 after birth, a few days after septation is normally thought to stop, DEX shows no effect either on the number of alveoli normally generated by $\mathrm{d} 28$ or on the normal volume of the average alveolus found at that age (Table 4). Moreover, the same number of alveoli found under both conditions (DEX and normal) is not simply due to a different combination of values in alveolar volume density and lung volume because no significant differences were found for these parameters between DEX-treated and untreated animals (Tables 1 and 2).

It also has been shown repeatedly that exposure of rats to HYP (>95\% oxygen) during the early postnatal period (age 4 to $14 \mathrm{~d}$ ) results in fewer and larger alveoli than those found in rats exposed to normoxia $(10,14)$. As with DEX treatment, these early life inhibitory effects on normal lung development and restructuring are permanent $(9-12)$. These findings, supported by lung microscopy studies showing stunted secondary septa development, can be interpreted as an indication that high oxygen exposure or DEX treatment inhibits the normal septation process in the developing lung. In the present study, we found that exposure to HYP after the so-called "normal period of septation" is over produces overtly similar inhibitory effects as it does during the first 2 postnatal wk. Hyperoxic exposure resulted in larger and less numerous alveoli than found in normally developed rats (Table 4). As previously discussed about DEX treatment, no significant differences were found in either lung volume or alveolar volume density between HYP and normoxia-exposed animals (Tables 1 and 2), indicating again that the difference found in alveolar number is a true difference and is not related 


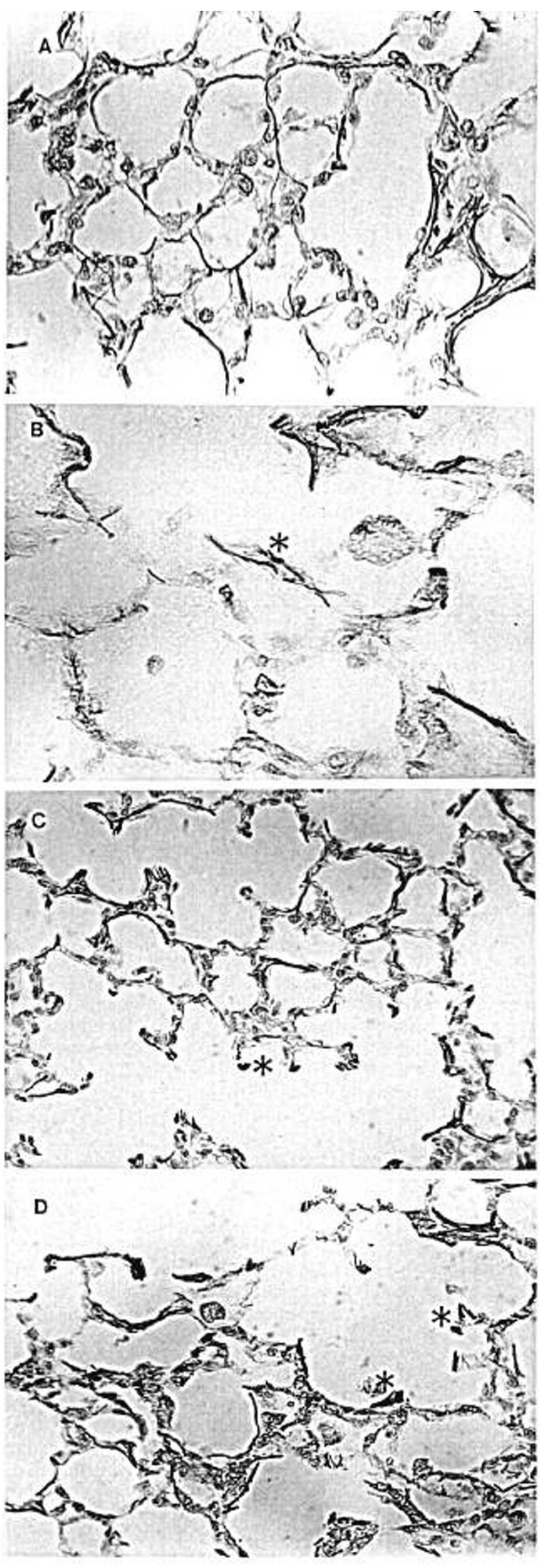

to different values for these two parameters, which could combine to generate the same estimated number of alveoli in both treatments.

Glucocorticosteroids are known to inhibit cell division in lung tissue (23), and because alveolar septa formation is the result of a process believed to require coordinated cell division, the administration of DEX during the first 2 postnatal wk was hypothesized by Massaro et al. (8) as a possible inhibitor of the formation of alveolar septa. Another reason that suggested the use of DEX to these investigators (8) was that the concentration of corticosterone in rat serum is normally quite low in rats during the first 2 postnatal wk (period of intense postnatal septation). Serum corticosterone then rather rapidly increases after this period when septation has been thought to normally be completed (24). Our finding of no inhibitory effect of DEX treatment [using similar doses as Massaro et al. (8)] when initiated at postnatal d 18 may be explained by the higher endogenous glucocorticoid levels by this age in the rat. Also, it may be that the lung tissue receptors for glucocorticoids either decline after approximately $2 \mathrm{wk}$ of age or become progressively desensitized by the elevated circulating levels of glucocorticoids.

The inhibitory effects on lung development by postnatal hyperoxic exposure are believed to be caused by the increased intracellular formation of reactive oxygen metabolites such as superoxide anion, hydrogen peroxide, and the hydroxyl radical. The hydroxyl radical has been shown to be an especially important DNA-damaging element (25). Intracellular formation of the hydroxyl radical depends on the concentration of intracellular ionic iron $(25,26)$, and in in vitro experiments, iron-chelating agents have been shown to inhibit both the formation of this cytotoxic radical and its damaging interactions with DNA (2527). Moreover, one of these iron-chelating agents, DFX, recently has been reported to substantially protect the lung of neonatal rats against the inhibitory effects of HYP on normal lung development (12).

Our treatment of HYP-exposed rats from postnatal d 18 to d 28 with DFX has shown that lung development is nearly normalized, compared with untreated HYP-exposed animals. DFX treatment during HYP resulted in a number of alveoli and a volume of the average alveolus that were not significantly different compared with air-exposed 28-d animal lungs (Table 4). Thus, DFX very effectively blocks the HYP inhibitory effect on lung alveoli formation both during the normal (age 3 to $13 \mathrm{~d}$ ) period and during the later period tested in this study (18 to 28 d of age), presumably by its effectiveness as an iron chelator preventing hydroxyl radical cytotoxicity.

Surface area of the gas-exchange region and surface area of the mouth opening of alveoli (Table 3 ) did not show any significant difference between conditions tested. These findings are in contrast to hyperoxic or DEX treatment during the early postnatal

Fig. 2. Comparative light-level micrographs of representative lung sections from air- and HYP-exposed rat pups age $28 \mathrm{~d}$ (exposure period age 18 to $28 \mathrm{~d}$ ). Orcein stain was used to emphasize elastin fibers. A. Air. original magnification $\times 160 ; B$, oxygen $\times 160 ; C$, air $\times 100$, and $D$, oxygen $\times 100$. Note the smaller and more homogeneous alveoli size in the air $(A)$ is the oxygen-exposed rat lungs $(B)$ and the more circumferential arrangement of the dark-staining elastin fibers. After HYP $(B)$, not only are the number of fibers reduced and the circumferential arrangement around the alveoli lacking, but the fibers themselves in the oxygen-exposed lungs appear either aggregated or frayed in areas $\left(^{*}\right)$. Note in the air lung at lower magnification $(C)$ the rather homogeneous small alveoli and the ordered way the mouths of the alveolar sacs come off the terminal airway or alveolar duct, with dense elastin delineating the mouths of each alveolar sac $\left(^{*}\right)$. In the oxygen-exposed lung $(D)$, the alveoli are basically larger and more heterogeneous in diameter and the mouths of the alveolar sacs where they come off the alveolar duct are much less ordered, with some areas of stunted development and other areas with more haphazard elastin deposition than in the air-exposed normal $28 \mathrm{~d}$ rat lung $\left({ }^{*}\right)$. 
period when each of these inhibitory agents substantially reduces the development of a normally much-expanded surface area of the gas-exchange region of the lung. The reason for this different behavior may be that although septation is an important factor in lung development during the first 2 postnatal wk, it may become less important as a primary mechanism for surface area enhancement after that age. Therefore, its inhibition during the earlier postnatal period has a more pronounced influence on surface area than at a later age.

Volume density of alveolar air was practically unchanged between treatments or age groups. The same was true for alveolar duct air volume density (Table 1). Therefore, the volume density of the gas-exchange air compartment, i.e. alveolar air volume plus alveolar duct air volume per unit lung volume, seems to be regulated in a way that is not altered by any experimental condition we tested.

The approximately $33 \%$ increase in the volume of the average alveolus and the doubling of the number of alveoli in normally developed rats between ages $13 \mathrm{~d}$ and $28 \mathrm{~d}$ (Table 4) suggests that lung gas-exchange surface area expansion in this later postnatal period occurs as a result of a combination of expansion of the volume of the average alveolus, replication of alveoli, and/ or septation. The greater volume of the average alveolus and lesser number of alveoli in the HYP-exposed animals can be explained by assuming that septation continues during the $3 \mathrm{rd}$ and 4 th postnatal wk in normal animals and is blocked by HYP because total alveolar volume was not found to be significantly different between treatments at $28 \mathrm{~d}$ of age (Table 2), and septation per se does not have any bearing on alveolar volume. Therefore, inhibition of septation will not alter total alveolar volume but must result in a bigger average alveolus and lesser number of alveoli, as was found. It could be argued that it is also possible that the inhibition was acting on the replication of alveoli rather than on septation, resulting in the same lesser number of alveoli. In this case, however, this would result in a smaller total alveolar volume (that was not found) unless an unlikely compensatory expansion acting simultaneously could bring alveolar number and volume of the average alveolus exactly to the values found.

Lung elastin development is believed to be an integral part of normal postnatal lung restructuring. The septa, which arise from the saccular walls and eventually merge to subdivide the larger saccules into smaller alveolar units, contain a conspicuous elastin component (28). Thus, any inhibitory influence that affects the normal septation process and normal alveolarization of the neonatal lung might be expected to also alter lung elastin development (and vice versa). It has been reported, for instance, that DEX treatment in the newborn rat inhibits normal elastin formation in the lung (29). Similarly, hyperoxic exposure has been reported to block lung elastin synthesis in the newborn (30). Thus, results of our morphometric quantitation of lung elastin in the 28-d-old rats exposed to HYP (Fig. 1) would further support the idea that septation is still actively proceeding in the lung beyond $14 \mathrm{~d}$ of age (and was markedly inhibited by high oxygen exposure beyond this age).

Although our elastic fiber values are given as relative length density (length per unit volume) rather than absolute total values, the comparison between groups seems appropriate in view of other data independently obtained. For example, volume density of alveolar air volume, alveolar duct air volume, and gas-exchange tissue volume as well as volume of total lung, alveolar air, alveolar duct air, and gas-exchange tissue (Tables 1 and 2) are not significantly different between groups. Also, previous application of the same technique to 14-d-old rats in air versus oxygen experiments compared with biochemical elastin determinations (elastin concentration in $\mathrm{mg} / \mathrm{g}$ dry lung) showed a remarkable parallelism between the two determinations: a $25 \%$ decrease biochemically versus $33 \%$ decrease morphologically in air-control versus oxygen control groups and a $13 \%$ decrease biochemically versus a $14 \%$ reduction morphologically in airtreated i'ersils oxygen-treated groups (31).

Finally, it seems important to note that a similar type of altered lung development (inhibited alveolarization and inhibited respiratory surface area expansion) found in neonatal animals exposed to HYP now has been reported in premature infants who developed bronchopulmonary dysplasia. Morphometric studies of the lungs of these infants who died of bronchopulmonary dysplasia after prolonged hyperoxic treatment have demonstrated a two-fold increase in mean linear intercept of alveoli, marked reductions in the number of alveoli (down to $25 \%$ or less of normal), and proportionate marked respiratory surface area reductions in comparison to age-matched infants $(32,33)$. Thus, in addition to causing well-characterized oxygen toxicity changes in the premature infant lung (edema, hemorrhage, hyaline membranes, fibrosis), prolonged high oxygen exposure could have important consequences on the development of respiratory reserve capacity in infants developing bronchopulmonary dysplasia. Whether concomitant DEX treatment of these infants exposed to high fractions of inspired oxygen to try to hasten their weaning from mechanical ventilation has additional important inhibitory effects on normal lung growth and development (as the animal studies caution) is presently unknown.

The main conclusions that can be derived from these studies would seem to be that septation does not stop abruptly at age 14 $\mathrm{d}$ in the rat; DEX treatment beyond age $14 \mathrm{~d}$ no longer has any demonstrable inhibitory effect on normal lung growth and development; exposure to HYP continues to result in a very marked inhibition of lung septation and alveolarization; and iron chelator treatment with DFX has a protective effect versus hyperoxic inhibition of normal lung development at both postnatal periods tested.

Acknowledgment. The authors thank Martha Sanchez for her patience in preparing the manuscript.

\section{REFERENCES}

1. Thurlbeck WM 1975 Postnatal growth and development of the lung. Am Rev Respir Dis 111:803-844

2. Burri PH 1974 The postnatal growth of the rat lung. III. Morphology. Anat Rec 180:77-98

3. Burri PH, Dbaly J, Weibel ER 1974 The postnatal growth of the rat lung. I. Morphometry. Anat Rec 178:711-730

4. Burri PH 1982 Reactive changes in pulmonary parenchyma after bilobectomy: a scanning electron microscopic investigation. Exp Lung Res 4:11-28

5. Blanco LN $1992 \mathrm{~A}$ model of postnatal formation of alveoli in rat lung. J Theor Biol 157:427-446

6. Wilson WL, Mullen M, Olley PM, Rabinovitch M 1985 Hyperoxia-induced pulmonary vascular and lung abnormalities in young rats and potential for recovery. Pediatr Res 19:1059-1067

7. Langston C, Kida K, Reed M, Thurlbeck WM 1984 Human lung growth in late gestation and in the neonate. Am Rev Respir Dis 129:602-613

8. Massaro D, Teich N, Maxwell S, Massaro GD, Whitney P 1985 Postnatal development of alveoli. Regulation and evidence for a critical period in rats. $\mathrm{J}$ Clin Invest 76:1297-1305

9. Blanco LN, Massaro GD, Massaro D 1989 Alveolar dimensions and number: developmental and hormonal regulation. Am J Physiol 257:L240-L247

10. Frank L 1991 Developmental aspects of experimental pulmonary oxygen toxicity. Free Radic Biol Med 11:463-494

11. Thibeault DW, Mabry S, Rezaickhaligh M 1990 Neonatal pulmonary oxygen toxicity in the rat and lung changes with aging. Pediatr Pulmonol 9:96-108

12. Frank L 1991 Hyperoxic inhibition of newborn rat lung development: protection by deferoxamine. Free Radic Biol Med 11:341-348

13. Sery Z, Keprt E, Obrucnik M 1969 Morphometric analyses of late adaptation of the residual lung following pneumonectomy in young adult rabbits. J Thorac Cardiovasc Surg 57:549-557

14. Frank L, Groseclose EE 1982 Oxygen toxicity in newborns: the adverse effects of undernutrition. J Appl Physiol 53:1248-1255

15. Scherle WA 1970 A simple method for volumetry of organs in quantitative stereology. Mikroskopie 26:57-60

16. Cruz-Orive LM, Weibel ER 1981 Sampling designs for stereology. J Microsc 122:235-257

17. Weibel ER 1979 Stereological Methods, Vol I. Academic Press, London, pp $9-196$

18. Cruz-Orive LM 1987 Particle number can be estimated using a disector of unknown thickness: the selector. J Microsc 145:121-142

19. Gundersen HJG 1986 Stereology of arbitrary particles. J Microsc 143:3-45 
20. Niewoehner DE, Kleinerman J 1977 Morphometric study of elastic fibers in normal and emphysematous human lungs. Am Rev Respir Dis 115:15-21

21. Duncan DB 1955 Multiple range and multiple $F$ tests. Biometrics 11:1-42

22. Kramer CY 1956 Extension of multiple range tests to group means with unequal number of replications. Biometrics 12:307-310

23. Morishige WJ, Joun NS 1982 Influence of glucocorticoids on postnatal lung development in the rat: possible modulation by thyroid hormones. Endocrinology 111:1587-1594

24. Henning SJ 1978 Plasma concentration of total and free corticosterone during development in the rat. Am J Physiol 235:E451-E456

25. Halliwell B, Gutteridge JMC 1989 The chemistry of oxygen radicals and other oxygen-derived species. In: Halliwell B, Gutteridge JMC, (eds) Free Radicals, in Biology and Medicine, 2nd Ed. Clarendon Press. Oxford. England. pp 22-85

26. Thomas CR, Aust SD 1989 Role of metals in oxygen radical reactions and oxidative stress. In: Miguel T, Quintanilha AT, Weber H (eds) Handbook of Free Radicals and Antioxidants in Biomedicine, Vol I. CRC Press, Boca Raton, FL, pp 37-48

27. Kvietys PR, Inauen W. Bacon BR. Grisham MB 1989 Xanthine oxidase- induced injury to endothelium: role of intracellular iron and hydroxyl radical. Am J Physiol 257:H1640-H1646

28. Noguchi A. Samaha H 1991 Developmental changes in tropoclastin gene expression in the rat lung studied by in situ hybridization. Am J Respir Cell Mol Biol 5:571-578

29. Schellenberg J-C, Liggins GC, Stewart AW 1987 Growth, clastin concentration. and collagen concentration of perinatal rat lung: effects of dexamethasone. Pediatr Res 21:603-607

30. Bruce MC. Pawlowski R. Tomashefski Jr JF 1989 Changes in lung elastic fiber structure and concentration associated with hyperoxic exposure in the developing rat lung. Am Rev Respir Dis 140:1067-1074

31. Frank L, McLaughlin, GM 1993 Protection against acute and chronic hyperoxic inhibition of neonatal rat lung development with the 21 -aminosteroid drug U74389F. Pediatr Res 33:632-638

32. Margraf LR. Tomashefski Jr JF. Bruce MC. Dahms BB 1991 Morphometric analysis of the lung in bronchopulmonary dysplasia. Am Rev Respir Dis $143: 391-400$

33. Sobonya RE, Logvinoff MM. Taussig L. Theriault A 1983 Morphometric analysis of the lung in prolonged bronchopulmonary dysplasia. Pediatr Res $16: 969-972$ 